\title{
A portable piezoelectric tactile terminal for Braille readers
}

\author{
Ramiro Velázquez*, Hermes Hernández and Enrique Preza \\ Mecatrónica y Control de Sistemas, Universidad Panamericana, Aguascalientes, Mexico
}

\begin{abstract}
This paper introduces a novel concept on reading assistive technologies for the blind: the TactoBook, a system that is able to translate entire electronic books (eBooks) to Braille code and to reproduce them in portable electronic Braille terminals. The TactoBook consists of a computer-based translator that converts fast and automatically any eBook into Braille. The Braille version of the eBook is then encrypted as a file and stored in a USB memory drive which is later inserted and reproduced in a compact, lightweight, and highly-portable tactile terminal. In particular, this paper presents a piezoelectric ultrasonic actuation approach to design and implement such portable Braille terminal. Actuating mechanism, design concept, first prototype, and performance results are presented and discussed.
\end{abstract}

Keywords: Assistive device, Braille, linear actuator, piezoelectric motor, tactile actuator (taxel), tactile terminal

\section{Introduction}

The 2011 World Health Organization (WHO) report on visual impairment and blindness indicates that there are 285 million people worldwide with some kind of visual impairment, 39 million of whom are totally blind [26].

This report estimates that approximately $90 \%$ of this population lives in low-income developing countries. This fact raises an economical barrier to purchase adequate equipment which is mostly sold in developed countries. Furthermore, the WHO predicts that the number of visually impaired will rise due to the large number of elderly people that will develop imminently some type of visual problem in the short/medium term.

The issue of the blind and visually impaired represents a serious challenge for governments, NGOs, health organizations, and society who will have to

*Corresponding author: Ramiro Velázquez, Mecatrónica y Control de Sistemas, Universidad Panamericana, Aguascalientes, Mexico. E-mail: rvelazquez@ags.up.mx. figure out the means to provide adequate health-care and support to enable the blind live independent and productive lives.

Sight is undoubtedly the most important human sense. It is considered fundamental for performing daily tasks such as crossing a street, looking for an address, or just reading a newspaper. For the blind, research on assistive systems has traditionally focused on two main areas: information transmission and mobility assistance. More recently, information transmission through computers and electronic information access have been important research and development (R\&D) fields.

The main problem related to computer access for the blind consists of reading any text displayed on a PC screen. Popular solutions are voice synthesizers, screen magnifiers, and Braille output terminals. Voice synthesizers practically read the computer screen; screen magnifiers enable on-screen magnification for those with low-vision, and Braille output terminals are plugged to the computer so that information on the screen is displayed in Braille [2]. 
In particular, typical Braille terminals consist of 80 cells. A Braille cell, which represents a single character, consists of six contact pins. A contact pin may be raised at any of the six positions to form 64 combinations (although some are omitted in practice because they feel tactually the same). Each combination conveys to the reader a letter, word, number, or symbol. There are also eight-dot cells that are used to replace a standard two-cell symbol (for example, when indicating an upper case letter). In any case, each Braille cell costs around 40 USD which makes the tactile terminal quite an investment.

Cost is not the only drawback when more ambitious applications are envisaged such as portable Braille terminals: 1) Dimensions of portable Braille terminals on the market today are $56.6 \times 2.2 \times 7 \mathrm{~cm} \mathrm{[9]} \mathrm{which} \mathrm{are}$ burdensome devices to carry. 2) Braille terminals are only output devices; they are computer dependent at all times.

This paper introduces the TactoBook system, a general purpose highly-portable Braille terminal that aims, in particular, to make eBooks accessible to the blind and visually impaired.

Industrial fabrication of tactile print books or Braille books is nowadays poor and their implementation is a challenging and slow manual process: each letter has to be translated into six contact points involving cutting, stapling, and gluing plastic point labels on a paper [16]. Coding even a small five-page text becomes a titanic effort. Unfortunately, the resulting Braille book is burdensome and uncomfortable to hold and carry, once read it is less interesting and sometimes it does not last very long with enthusiastic use, especially by children.

Braille printers do exist. However, the result is the same in terms of bulkiness and the simplest ones cost around 3000 USD [5].

On the other hand, the eBook publishing industry is rapidly growing. Since the Internet revolution, the 400year old publishing industry had no choice but to move to the online space in order to satisfy their also growing audience. In consequence, nowadays regular print books from every major publishing house are commonly found in electronic format and the current trend is that more and more are digitized and uploaded to the Internet.

This paper presents a prototype system that intends to overcome the accessibility problems of tactile print books by offering a simple, fast, and automatic translation to Braille, possibility of multiple use (a wide number of eBooks can be stored and reproduced using the same device) while being robust and portable.

The rest of the paper is organized as follows: Section 2 introduces the TactoBook system and overviews its main modules. Section 3 focuses on the tactile terminal and describes its actuation approach, characterization, and experimental evaluation. Section 4 presents the integration approach and the first prototype of portable Braille terminal developed. Finally, Section 5 concludes summarizing the main contributions and future work perspectives.

\section{The TactoBook system}

Figure 1 shows the TactoBook concept [22]: using standard computational resources, an eBook is translated to Braille code, encrypted as a file, and stored in a regular USB pen drive. This memory drive is then extracted from the computer and inserted into a compact, lightweight, and highly-portable tactile terminal where the file is opened, processed, and reproduced in a set of 10 Braille cells at a time. By touching the pins, the user is able to read the eBook. Note that this concept has a twofold purpose:

(1) to be an eBook portable reading device for the blind in which case, there is no computer dependency and

(2) to serve as a general purpose output Braille terminal in which case, there is computer dependency.

\subsection{Braille translator}

There are two grades of text-to-Braille transcription. The basic transcription is called Grade 1 Braille and it is a direct substitution of normal print letters for letters from the Braille alphabet. Grade 2 Braille is the advanced transcription in which a single cell can represent a shortened form of a word. Official Grade 2 Braille has 189 contractions. A complex system of styles, rules, and usage has been developed for this grade to make reading and writing Braille much faster and shorter. However, Grade 2 transcription can only be done properly by a human [3].

The TactoBook translator is a computer-based tool that converts a text file into Braille code using Grade 1 Braille. It basically consists of a lookup table where each letter is associated to its Braille equivalent. 


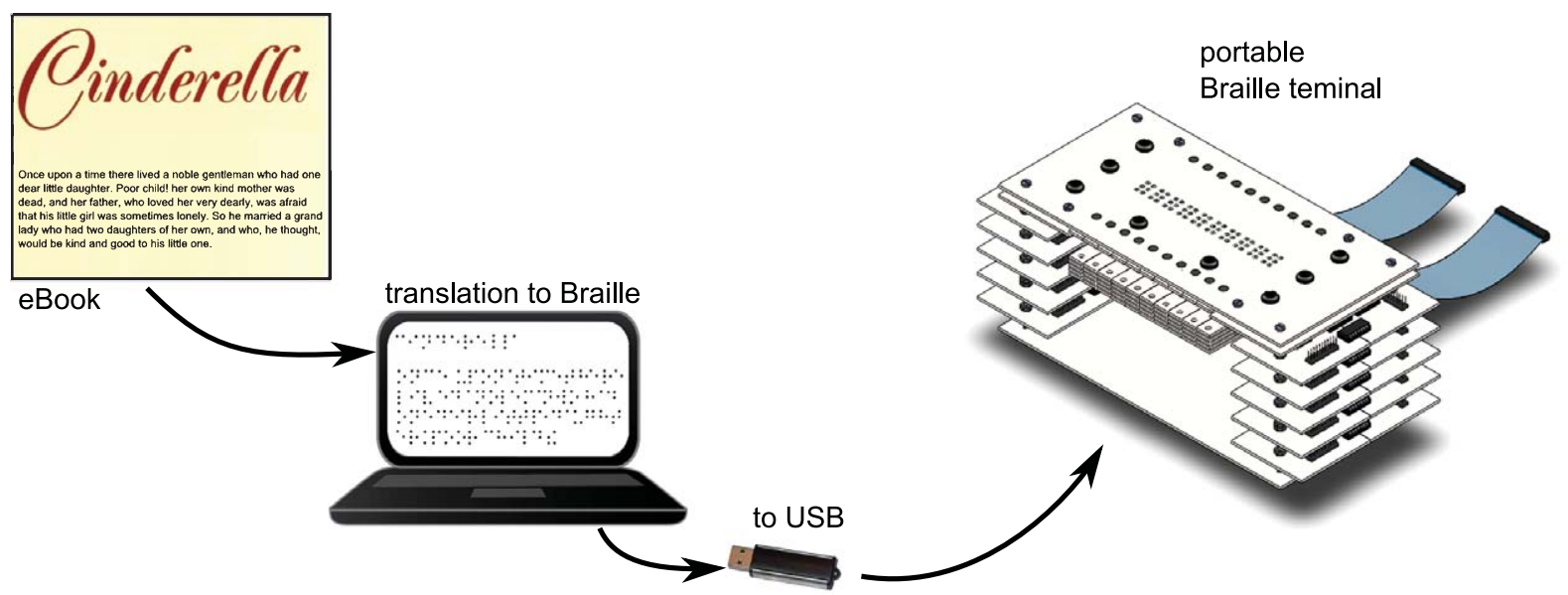

Fig. 1. The TactoBook concept: eBooks in portable Braille terminals.

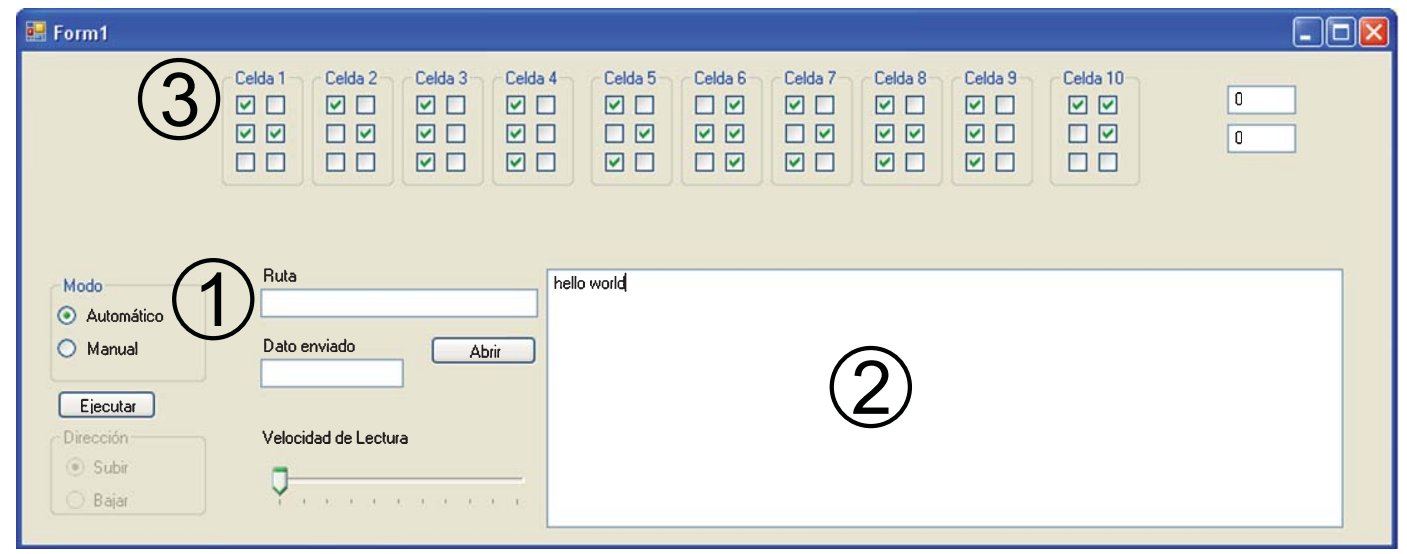

Fig. 2. Snapshot of the TactoBook Braille translator and its 3 main functions: (1) file upload, (2) textbox, and (3) manual mode.

Figure 2 shows a snapshot of the user interface. Its architecture and operative code, developed under a Visual.Net environment, were conceived in order to offer great flexibility and simplicity in data transcription:

(1) File-upload function: This function allows the user to upload any supported file to be translated into Braille. For the moment the interface supports the most common file-formats such as portable document files (pdf), unformatted text files (txt), and Word document files (doc). Current work focuses on Internet file-formats, such as Extensible Markup Language (XML).
(2) User-defined text entry function: This feature allows the user to enter any text to be translated into Braille either by typing it or just by pasting it into a textbox.

(3) User-defined pin control function: This function allows the user to manage the position status (up or down) of any pin from the 10-cell-array so that the user can display any desired pincombination. This could be helpful to display a user-customized Braille codification.

Experimental measurements of the translator's computing time show a quite linear performance: using a standard computer with $256 \mathrm{MB}$ of RAM memory, a 
page of 2000 characters is translated in approximately $0.8 \mathrm{~s}$. Roughly, a standard text book of 300 pages can be fully translated to Braille in $240 \mathrm{~s}$ [23]. The equivalent tactile print book would consume approximately 1800 pages.

Braille translation is in fact a very challenging issue. Braille software translators have to comply with three main features: standardization, appropriate contexttranslation, and navigability.

(1) Standardization: Nowadays, several Braille software translators can be found in the market $[4,18]$. On one hand this allows to choose among many different file-formats that comply with practically any requirement, but on the other hand it causes compatibility issues because, as always, not any software is designed to work with any hardware. This is undoubtedly a technical barrier for file interchange and file access.

(2) Context-translation: Most Braille translators are intended to work properly with a few texttypes, such as novels, essays, or only-text files but will have bad performance when trying to translate, for example, a scientific article containing images and mathematical equations. The main reason is that the translation process is not always straightforward, but is, in many cases, context-specific. To avoid these problems the user would have to choose the translationprotocol for every text-type to be translated in advance or the software would have to determine by itself the appropriate translation-protocol and file-type, which, at this time, seems quite far.

(3) Navigation: Another related problem arises when trying to establish navigation-standards that would allow the user to freely surf Braille- files. This could be very helpful to the user, since it would provide him important information about his location at the file, for instance: chapter, page, and paragraph-number and will also permit him to move forward, backward, or jump to a specific location. One standard that has proven moderate success is the DAISY/NISO [1] used nowadays for audio-books. It basically splits a file into hierarchies: chapters, pages, paragraphs, lines, etc., adding markups at every point which work as reference-guides for the user. Nevertheless, it has not been totally applied to Braille. This standard has been found to be a good solution to navigate among and along the TactoBook's files. Of course, the proper codification for navigation is saved in a Braille-file as well, making the file less compatible with other hardware.

\subsection{Braille terminal}

Most tactile devices commercially available are piezoelectric Braille terminals. In the standard Braille configuration, pins are spaced $2.54 \mathrm{~mm}$ apart and produce vertical strokes of $0.7 \mathrm{~mm}$. Each pin delivers a $170 \mathrm{mN}$ pull force at a refreshable bandwidth of $6 \mathrm{~Hz}$.

Braille terminals have changed little in the past 25 years: they are based on piezoelectric bimorph benders and relay-lever mechanisms [27]. For piezoelectric based displays, the bimorph bender produces vibrations that move the pin up and down when placed in an electric field (Fig. 3a). For relay-lever mechanisms, when voltage is supplied, the relay pushes the pin up; when the power is off, the self-weight of the lever mechanism lets the pin downward to its original position (Fig. 3b).

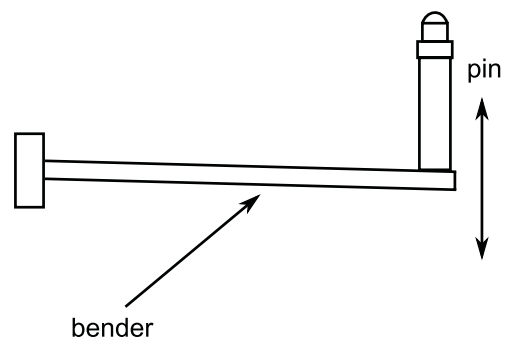

(a) Piezoelectric bimorph bender.

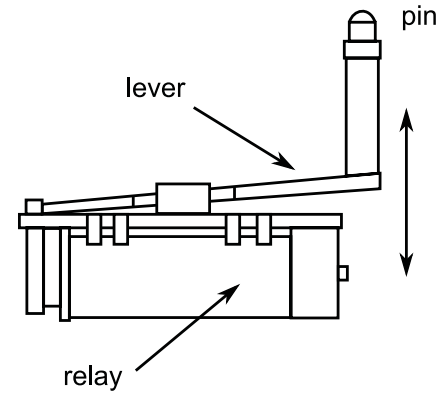

(b) Relay-lever system.

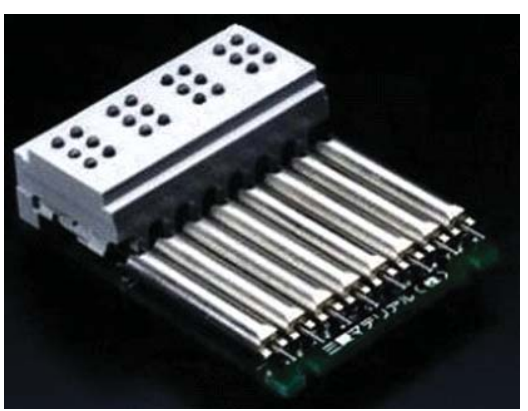

(c) Physical implementation

Fig. 3. Examples of existing Braille mechanisms. 
Both mechanisms share a common design drawback: benders and relay-lever systems are implemented as an additional burdensome module attached to the contact pins that may compromise integration capacity (Fig. 3c). In consequence, Braille terminals are not usually portable.

In the last decades, many tactile devices, all sharing the principle of raising individual pins, have been developed using a wide range of technologies. Approaches range from traditional actuation technologies such as servomotors [25], electromagnetic coils [6], piezoelectric ceramics [19], and pneumatics [24] to the new ones: shape memory alloys (SMAs) [15], electroactive polymers (EAPs) [11], electrorheological (ER) fluids [20] and micro-electromechanical systems (MEMS) [13]. While promising, these technologies have yet to prove significant advantages (cost and performance) to replace the present piezoelectric approach.

An optimal Braille terminal for the TactoBook system should accomplish the following design criteria:

(1) It should comply with Braille standards.

(2) It should be portable. It is desirable that the Braille terminal is as lightweight and small as possible and can easily fit into a small pack. Furthermore, it suggests an unobtrusive and inconspicuous device, which are essential for blind people.

(3) It should be energy efficient. The power source faces two constraints: supply considerable lasting energy to the tactile terminal for a regular journey and be lightweight as it must respect the portability criterion.

(4) The tactile terminal global design should be robust, maintainable, and simple/fast/low-cost to fabricate to represent a significant advantage over commercially available Braille terminals and for future industrial production.

Sections 3 and 4 will focus on the design and implementation of the Braille terminal.

\section{Actuation approach}

\subsection{Structure and principle}

To overcome the compactness problem of traditional Braille actuators, we focused our attention on piezoelectric linear motors. In particular, in a tiny ultrasonic linear actuator (TULA) from Piezoelectric Technology Co [17].

This actuator consists of a shaft, a mobile element or slider, and a piezoelectric ceramic disk (Fig. 4a).

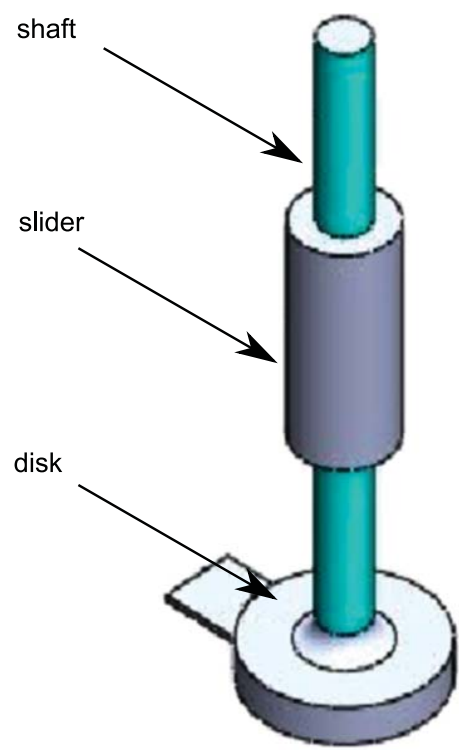

(a) Conceptual design.

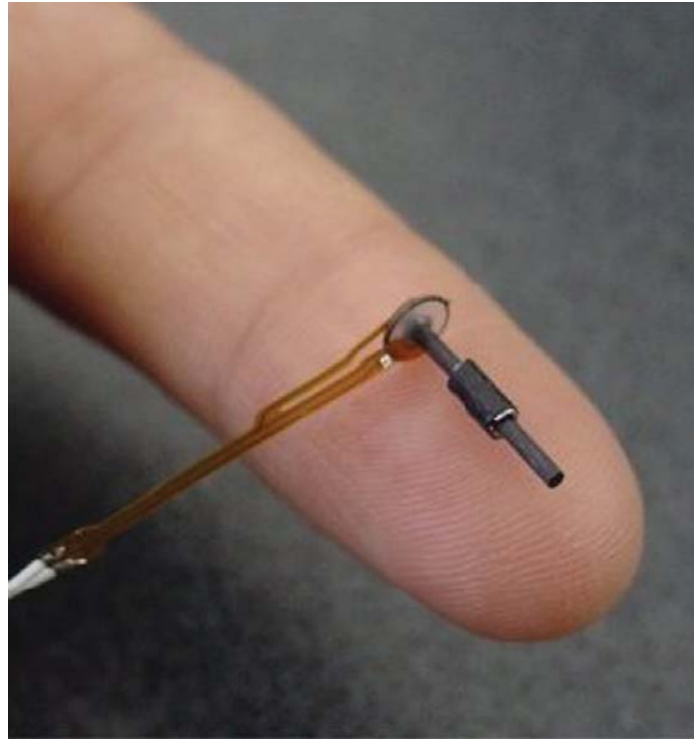

(b) TULA-35 prototype from Piezo-Tech Co.

Fig. 4. Miniature piezoelectric ultrasonic linear motor. 


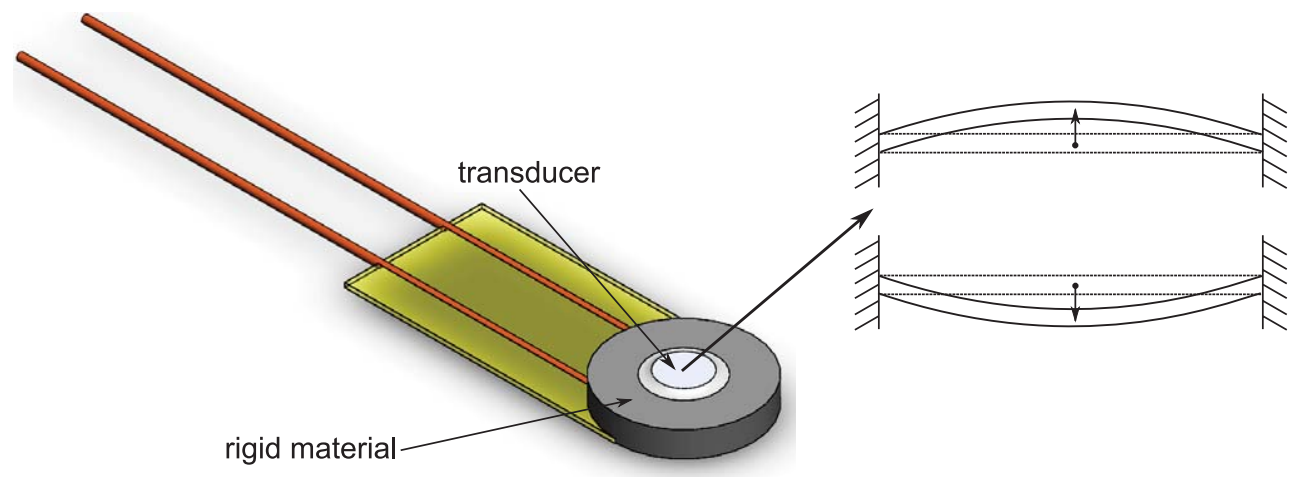

Fig. 5. Structure of the piezoelectric ceramic disk and vibration of the transducer.

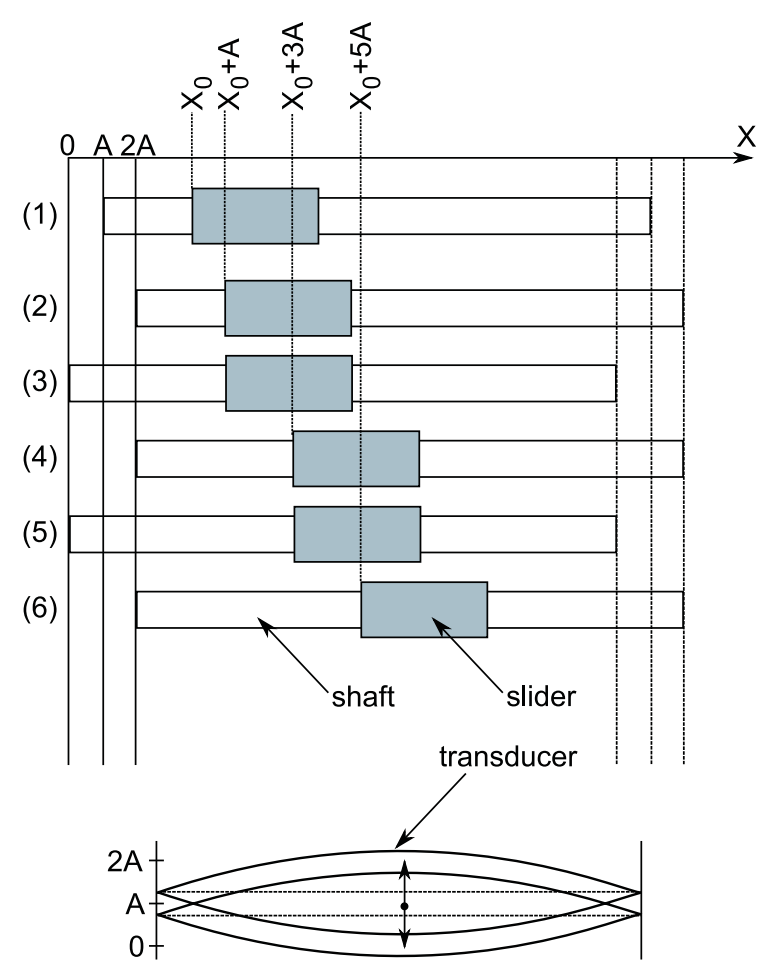

(a) The principle of inertia displacement.

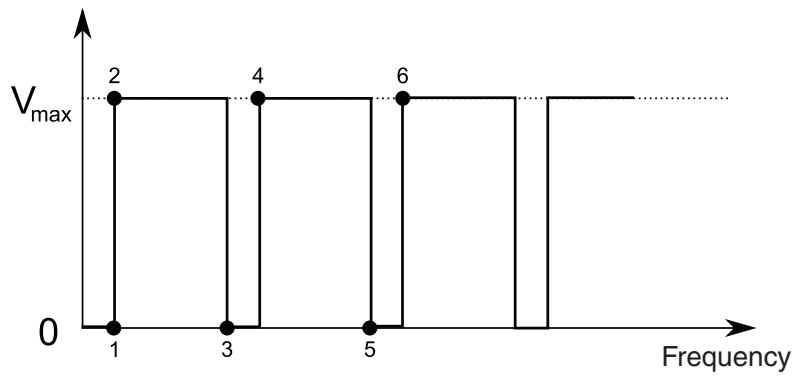

(b) The square wave driving electrical signal. A duty cycle of $80 \%$ is typically used for driving the piezo-motor.

Fig. 6. Operation principle for the piezoelectric ultrasonic linear motor.

It is miniature and ultra-lightweight: $2.4 \mathrm{~mm}$ diameter, $11.6 \mathrm{~mm}$ length, and $370 \mathrm{mg}$ mass (Fig. 4b). Note that the entire actuator is tightly packed in its $2.4 \mathrm{~mm}$ diameter with no burdensome benders or relay-lever mechanisms.

The ceramic disk consists of a rigid part and a transducer. When voltage is applied, the transducer vibrates creating upward/downward fluctuations which are transfered to both shaft and slider (Fig. 5).

Its linear motion is based on the principle of inertia displacement by square wave electrical potential [10].

This operation principle is shown in Fig. 6a: (1) at the initial position, the motor is not energized, the shaft is in position $A$ and the slider is located in position $x_{0}$. 
(2) When maximum voltage is applied, both shaft and slider move together a distance $A$ (i.e. shaft to position $2 A$ and slider to position $x_{0}+A$ ). (3) As voltage falls to zero, the shaft slips backwards a distance $2 A$. However, the slider remains in its position because of its inertia. (4) When voltage increases to its maximum, both shaft and slider move again together a distance $2 A$ (i.e. shaft to position $2 A$ and slider to position $x_{0}+3 A$ ). The cycle repeats: (5) voltage decreases, shaft goes back to 0 while the slider remains in $x_{0}+3 A$. (6) Voltage increases, the shaft goes to position $2 A$ and the slider moves to position $x_{0}+5 A$.

Figure $6 \mathrm{~b}$ shows the corresponding square wave driving electrical signal.

Figure 6 shows the slider moving from left to right. Reverse motion can be achieved by inverting the duty cycle of the square wave electrical potential.

\subsection{Tactile actuator}

This piezo-motor has been used to design and develop a novel tactile actuator $\left(\operatorname{taxel}^{1}\right)$. This taxel is shown in Fig. 7. Note that a plastic contact pin has been simply attached to the slider.

The taxel is bi-stable: due to friction between the slider and the shaft, the slider is capable of retaining its position without any power. Energy is only needed when changing from one position to another. Bi-stability avoids power consumption, useless output work, and a gradual degradation of the actuator's performance.

\subsection{Modeling and simulation}

Piezoelectric ceramics are a special type of dielectric materials that are known for what are called the piezoelectric and reverse piezoelectric effects. The piezoelectric effect causes a dielectric to produce an electrical potential when it is subjected to mechanical vibration. In contrast, the reverse piezoelectric effect causes the dielectric to produce vibration when it is placed in an electric field.

Figure 8a shows a schematic representation of both effects. Naturally, our interest focuses on the reverse piezoelectric effect. In this one, a voltage is produced in the dielectric when it is placed in an electric field. Due to the piezo-effect, the voltage generates a transduced force which, through a mechanical relation, results on an elongation.

\footnotetext{
${ }^{1}$ Taxel: from tactile pixel.
}

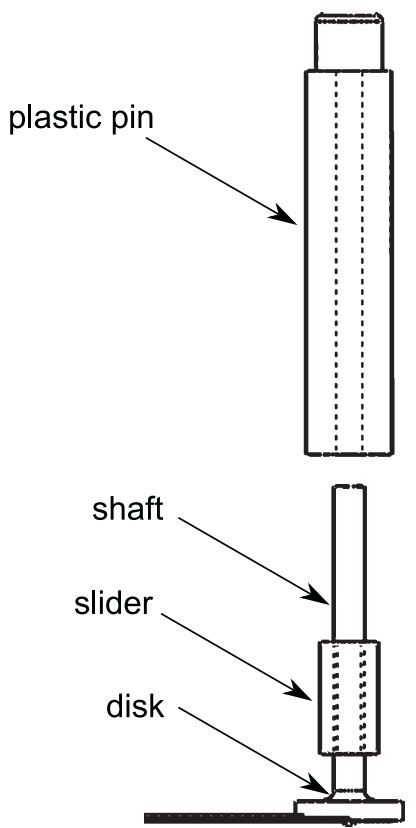

Fig. 7. Structure of the taxel.

A fairly accurate overall electromechanical model of both effects has been proposed in [7]. Figure $8 \mathrm{~b}$ shows the simplified model for the reverse piezoelectric effect.

Here, the electrical model is basically represented by an RC circuit. The piezo effect is represented by $T_{e m}$, which is an electromechanical transducer with transformer ratio $T_{e m}$. The voltage $u_{p}$ is due to the piezo effect. The force $F_{p}$ is the transduced force from the electrical side and mechanically drives the piezo material. The resulting elongation of the piezoelectric actuator is denoted by $y$. The mechanical relation between $F_{p}$ and $y$ is denoted by a simple mass-springdamper system.

For the taxel, the mechanical relational can be adapted as shown in Fig. 8c. Here, the mass $m_{1}$ moved by the transducer is the shaft while mass $m_{2}$ is the sum of the masses of the slider and plastic contact pin. The damping ratio $b_{1}$ is due to the transducer, $b_{2}$ is the viscous friction between the shaft and slider, and $k$ is the equivalent stiffness.

The complete set of electromechanical equations for the piezoelectric taxel is as follows:

$$
\begin{aligned}
& \frac{U_{p}(s)}{U(s)}=\frac{1}{R C s+1} \\
& F_{p}(s)=T_{e m} U_{p}(s)
\end{aligned}
$$




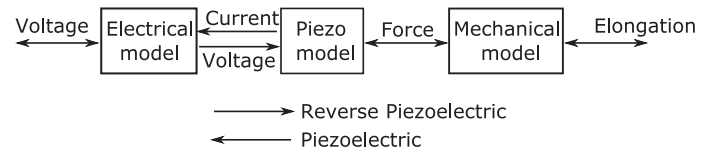

(a) Block diagram of the piezoelectric and reverse piezoelectric effects.

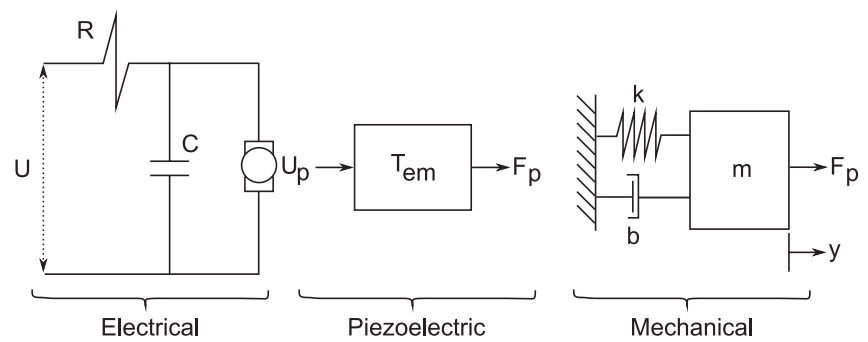

(b) Simplified electromechanical model for the reverse piezoelectric effect [7].

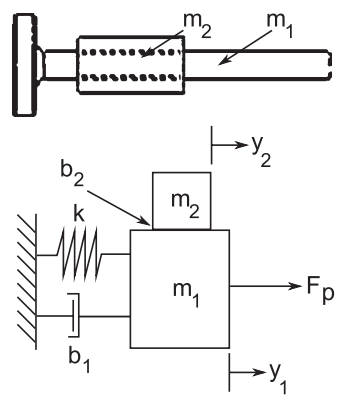

(c) Mechanical model for the taxel.

Fig. 8. The reverse piezoelectric effect and mechanical model for the taxel.

Table 1

Summary of the parameters involved in the taxel's modeling.

\begin{tabular}{lcc}
\hline Parameter & Value & Unit \\
\hline Resistance $R$ & 340 & $\Omega$ \\
Capacitance $C$ & $1.7 \times 10^{-9}$ & $\mathrm{~F}$ \\
Transducer ratio $T_{e m}$ & 0.0075 & $\mathrm{~N} / \mathrm{V}$ \\
Mass $m_{1}$ & $10 \times 10^{-6}$ & $\mathrm{~kg}$ \\
Mass $m_{2}$ & $500 \times 10^{-6}$ & $\mathrm{~kg}$ \\
Damping ratio $b_{1}$ & $1 \times 10^{-6}$ & $\mathrm{~N}-\mathrm{s} / \mathrm{m}$ \\
Viscous friction $b_{2}$ & $10 \times 10^{-6}$ & $\mathrm{~N}-\mathrm{s} / \mathrm{m}$ \\
Stiffness $k$ & $10 \times 10^{7}$ & $\mathrm{~N} / \mathrm{m}$ \\
\hline
\end{tabular}

$$
\frac{Y_{2}(s)}{F_{p}(s)}=\frac{b_{2} s}{\left[m_{2} s^{2}+b_{2} s\right]\left[m_{1} s^{2}+\left(b_{1}+b_{2}\right) s+k\right]-b_{2} s^{2}}
$$

Experimental and numerical procedures were developed to determine these parameters for the taxel. Values obtained are given in Table 1.

Figure 9 presents the electromechanical relations for the piezoelectric taxel. Figure 9a shows the evolution of voltage $u_{p}$ when a square wave input voltage $u$ of $15 \mathrm{~V}$ at $75 \mathrm{kHz}$ with $80 \%$ of duty cycle is applied. Note that the electrical behavior of the piezoelectric motor is effectively that of a high-frequency capacitor.
Figure $9 \mathrm{~b}$ shows the relation between voltage $u_{p}$ and transduced force $F_{p}$. Note that the actuator is capable of delivering pull forces of $120 \mathrm{mN}$.

Figure 9c shows the temporal evolution of the sliderpin displacement $y_{2}$. Note that the $75 \mathrm{kHz}$ transduced force $F_{p}$ is capable of moving slider and plastic contact pin a distance of $11.6 \mathrm{~mm}$ (the full stroke) in $0.64 \mathrm{~s}$. Note that, contrary to the operation principle shown in Fig. 6a, the slider actually slips backwards when moving along the shaft. However, this backwards distance is negligible compared to the forward displacement (Fig. 9c inset).

\subsection{Experimental evaluation}

Experimental procedures were conducted to determine the conditions that allow to obtain the best performance from the piezoelectric taxel.

In particular, it is of much interest to determine the relation between the voltage and frequency values of the input square signal and the output speed of the taxel.

For this purpose, a square wave voltage signal with $80 \%$ of duty cycle was used. Voltage was gradually increased from 8 to $24 \mathrm{~V}$ in steps of $2 \mathrm{~V}$. Frequency was gradually increased as well from 38 to $110 \mathrm{kHz}$ in steps 


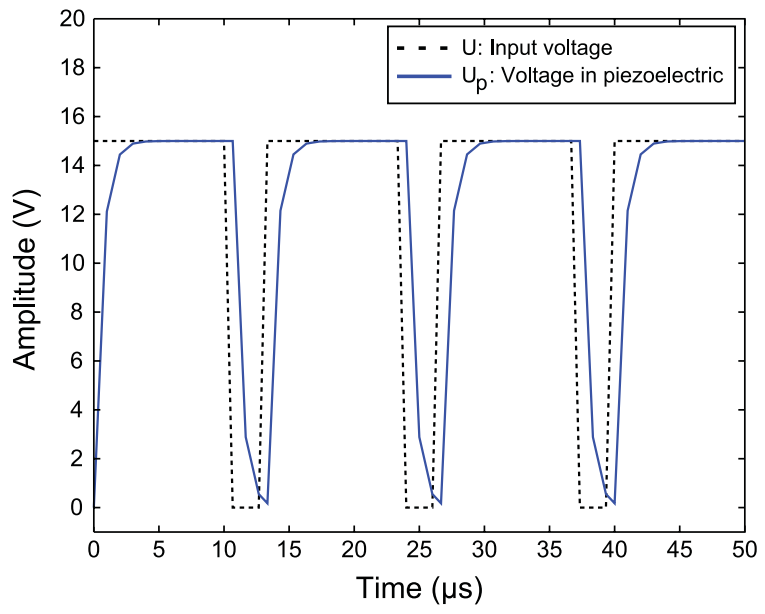

(a) Electrical simulation for the taxel.

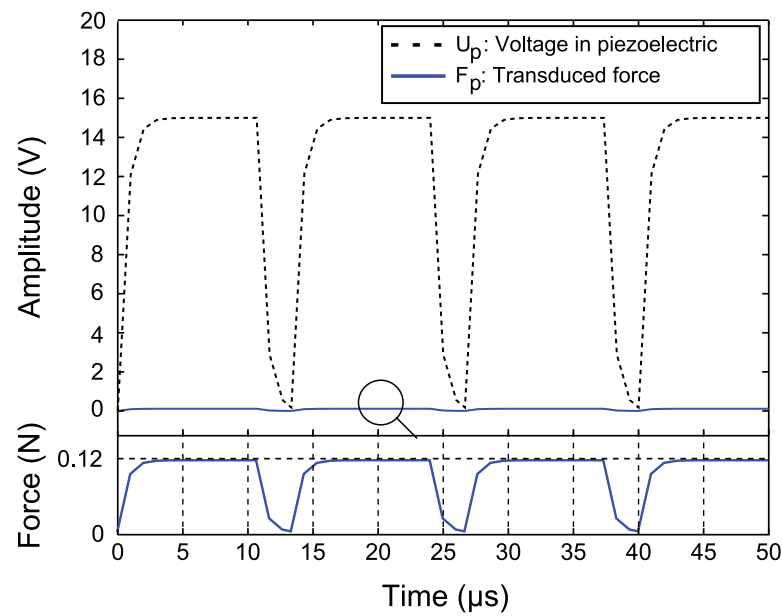

(b) Piezoelectric simulation for the taxel.

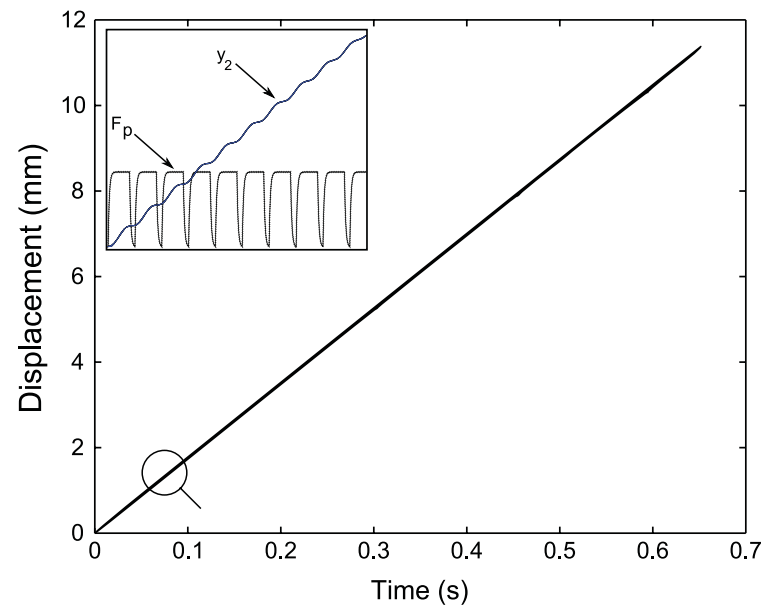

(c) Mechanical simulation for the taxel.

Fig. 9. Numerical simulation relations for the piezoelectric taxel.

of $2 \mathrm{kHz}$. This combination produces 333 speed samples that are largely enough to characterize the taxel's behavior.

Figure 10a shows the taxel's behavior in the speedfrequency-voltage space. To understand this surface, let us consider first the relation between speed and frequency. Figure $10 \mathrm{~b}$ shows this relation for a set of input voltages.

Note that all curves are morphologically the same and that there is a dead zone between $56-62 \mathrm{kHz}$. Contrary to the expected statement: "more voltage, more speed", the curve reveals that the maximum speed is developed at $16 \mathrm{~V}$. Note, for example, that approximately the same speed is developed at $12 \mathrm{~V}$ and $20 \mathrm{~V}$. Then, it is no worth to supply more than $16 \mathrm{~V}$ to the actuator. Finally, the curve reveals two operating zones: $44-56 \mathrm{kHz}$ and $62-110 \mathrm{kHz}$. Note that minimum 16 $\mathrm{V}$ are needed to drive the actuator in the $44-56 \mathrm{kHz}$ range. Lower voltages do not produce any response within this range.

Let us consider now the relation between speed and voltage. Figure 10c shows this relation for a set of input frequencies. Note a good performance between the 70$90 \mathrm{kHz}$ range. Again, for frequencies below $60 \mathrm{kHz}$, there is only response for voltages above $16 \mathrm{~V}$. Finally, note that above $16 \mathrm{~V}$, the taxel's performance worsens considerably.

From this analysis, it can be established that the best performance is obtained when supplying the taxel input square wave signals within 14-16 V and 70-80 kHz. 


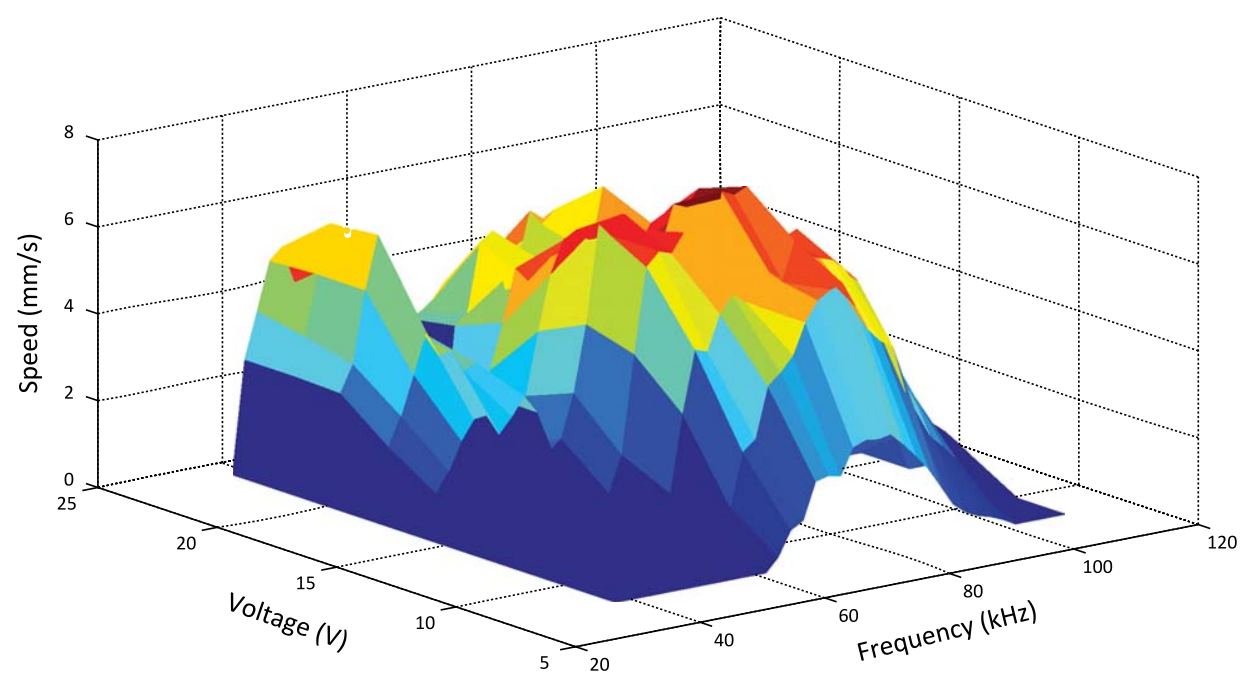

(a) Experimental behavior in the speed-frequency-voltage space.

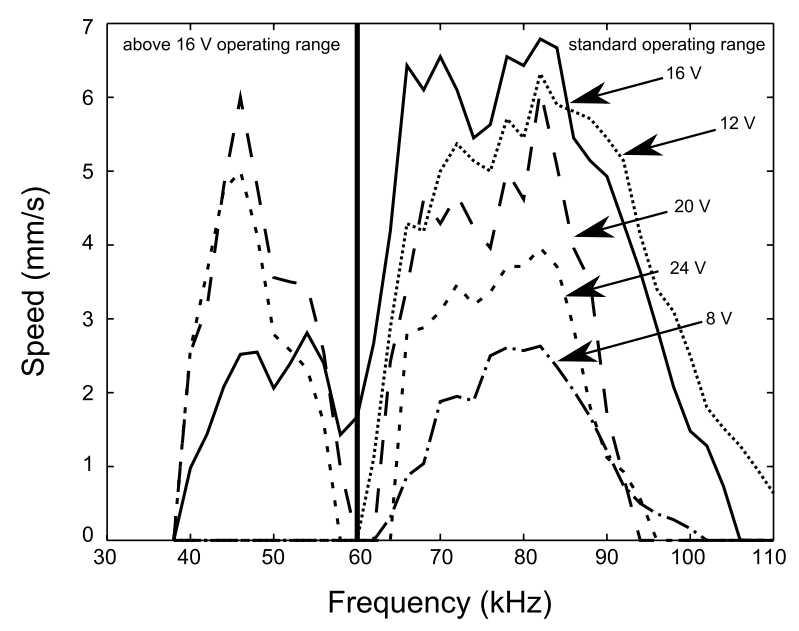

(b) Experimental speed-frequency curve.

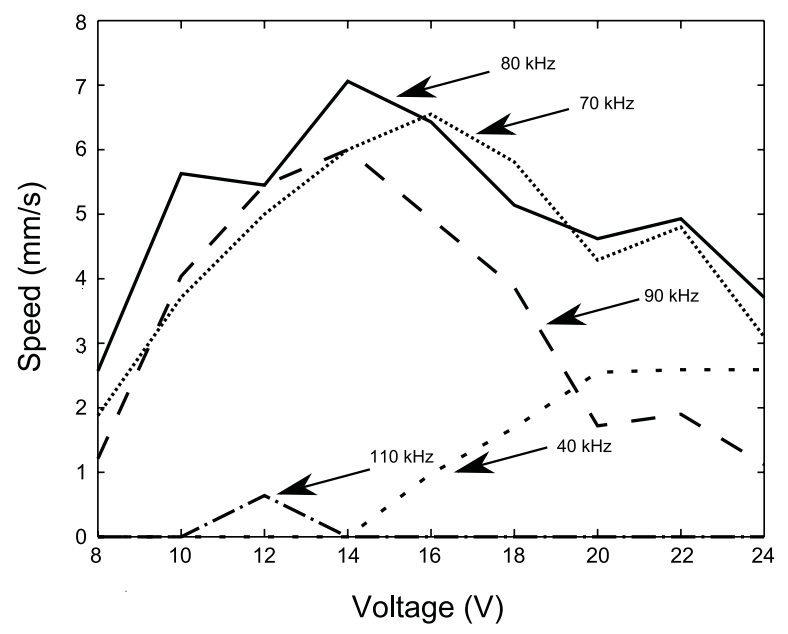

(c) Experimental speed-voltage curve.

Fig. 10. Experimental relations for the piezoelectric taxel.

Experimental procedures were also conducted to determine the pull force developed by the taxel. In particular, the relation between speed and load gives significant information on the performance to be expected when touching the pins.

For this purpose, a load was attached to the contact pin simulating the force applied by fingertips. Load was gradually increased from 6 to $120 \mathrm{mN}$ in steps of $6 \mathrm{mN}$. Speed was measured at optimal conditions of square wave input signal $(15 \mathrm{~V}, 75 \mathrm{kHz})$.

Figure 11 shows the results obtained. As any other actuator, speed decreases upon load. A survey of psychophysical experimental data indicates that a 50 to $100 \mathrm{mN}$ force is typically applied during fine-touch exploration [14]. Traditional Braille cells are designed to resist $170 \mathrm{mN}$. Simulation results predicted $120 \mathrm{mN}$ but the taxel can actually deliver $108 \mathrm{mN}$ pull forces.

\section{First prototype}

\subsection{Integration approach and prototype}

To implement the TactoBook Braille terminal, 60 of these piezoelectric taxels have to be integrated in 


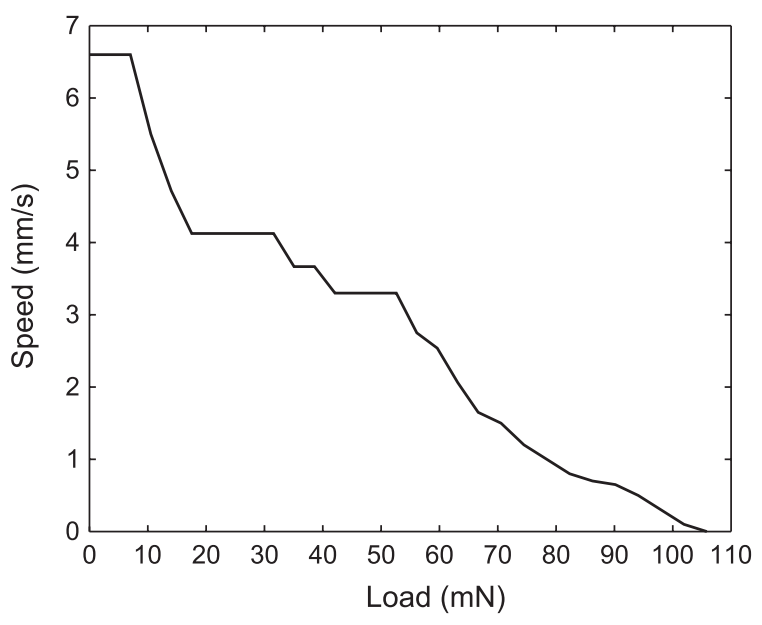

Fig. 11. Experimental speed-load curve.

a compact device. Main design goals of the Braille terminal are as follows:

(1) Taxels are to be independently addressed and their motion should not jam or limit the motion of neighbor taxels.

(2) Each taxel must be placed within a Braille cell so that it has convenient access to electric power without disturbing other taxels.

(3) A taxel should be easily removed if necessary.

The design concept finally retained to build the tactile terminal is based on a multi-layer approach: placing the actuators one next to the other in a single layer would result in a complex handling process. By using a multilayer design, the taxels can be properly placed to ease their handling/assembly/replacement.

Figure 12a shows the conceptual representation of a single Braille cell with the multi-layer approach [8]. This first design consists of seven layers: the first layer is the contact surface, the second layer serves as guiding structure of the contact pins. Layers 3 to 6 contain the taxels. Layers 3 and 4 contain two taxels each, while layers 5 and 6 only one each. Layer 7 serves as the base of the cell. Figure 12a inset details the structure of layers 3 to 6 . Note that six taxels are placed in different layers. Figure $12 \mathrm{~b}$ shows a prototype Braille cell built using this multi-layer design concept.

Ten of this cells integrate the TactoBook tactile terminal. Figure 13 shows both design concept and first prototype developed. Note that the electronic drive consists of a set of insertable boards, which are located inside the device. Each circuit board drives one Braille cell. Unfortunately, with off-the-shelf circuitry, the electronic drive is the most burdensome item of the terminal. Surface mount technology (SMT) components could be used to reduce board dimensions.

The overall performance of this first 10-cell prototype is quite similar to the one obtained with traditional 18-cell commercially available portable Braille terminals: controllable strokes along the $11.6 \mathrm{~mm}$ long shaft, $108 \mathrm{mN}$ pull force, and $5 \mathrm{~Hz}$ refreshable bandwidth when the stroke is set to $0.7 \mathrm{~mm}$, the Braille standard. The great advantage of this prototype over existent Braille terminals is that its structure is simpler, more compact, and lightweight: the full terminal is $1.1 \mathrm{~kg}$

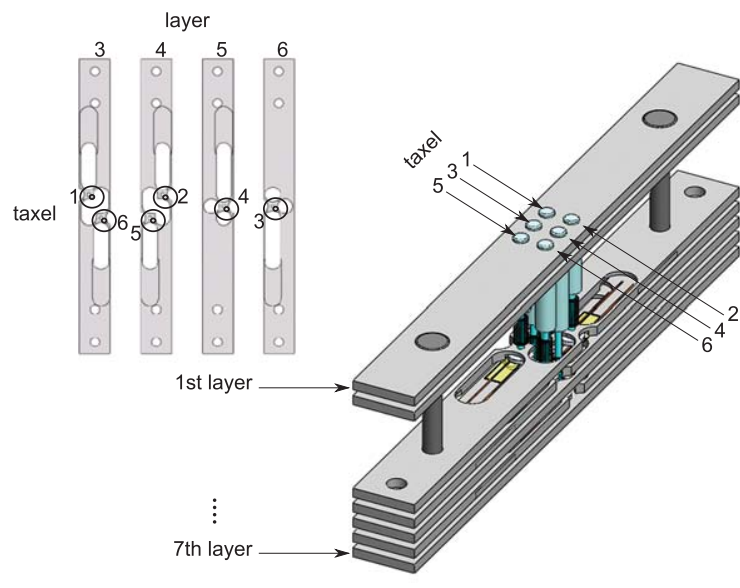

(a) Multi-layer approach and detail of the layers' structure.

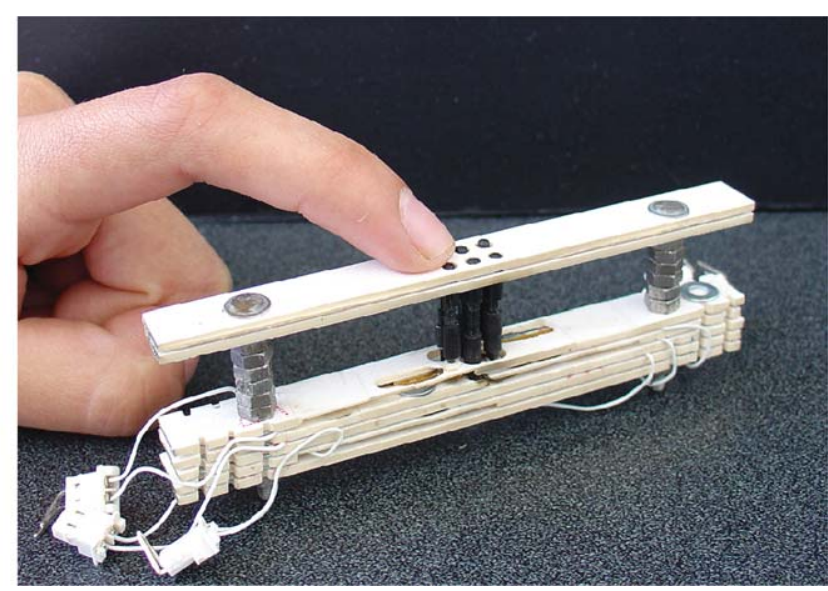

(b) Braille cell prototype.

Fig. 12. Design and implementation of a Braille cell with piezoelectric ultrasonic linear motors and a multi-layer approach. 


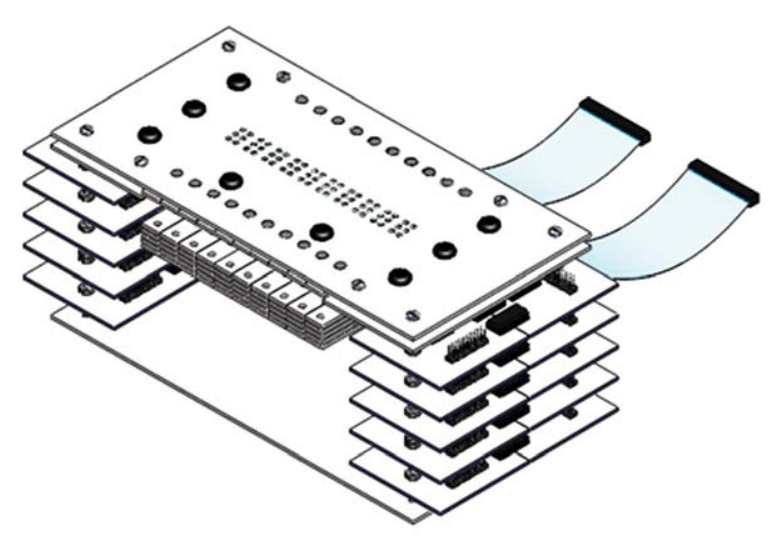

(a) Conceptual representation.

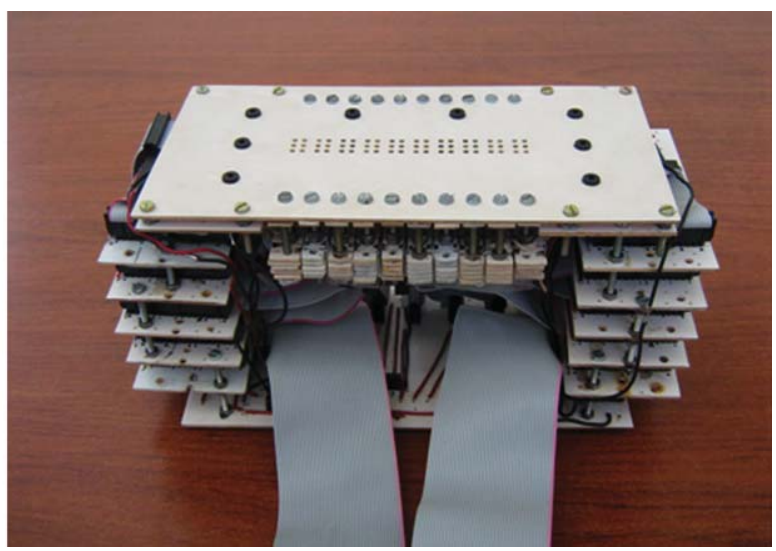

(b) First prototype.

Fig. 13. The TactoBook's first prototype of portable Braille terminal.

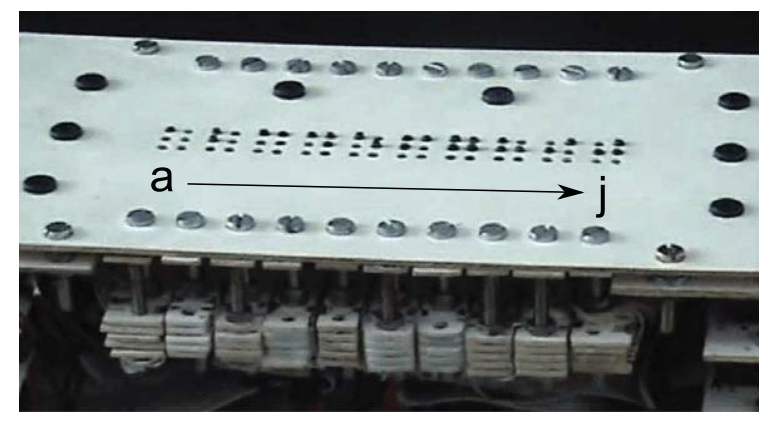

Fig. 14. An example of ten Braille characters (abcdefghij) displayed using the Braille terminal.

mass and its compact dimensions $(20 \times 15 \times 10 \mathrm{~cm})$ make it easily carried by the user.

Figure 14 shows an example of Braille characters displayed using this prototype.

\subsection{Drive system}

The general diagram of the electronic drive architecture that controls the 60 piezoelectric taxels is presented in Fig. 15a. As aforementioned, to move optimally the slider upward/downward along the shaft, a $15 \mathrm{~V}$ square signal at $75 \mathrm{kHz}$ is needed. This signal is applied to one terminal of the ceramic transducer while its inverse to the other. To achieve this, a microcontroller generates a $5 \mathrm{~V}, 75 \mathrm{kHz}$ square wave signal which is later amplified to $15 \mathrm{~V}$. Selection of the desired motors is also performed by the microcontroller.

In order to obtain the best performance of each motor, they must be supplied by its own driver that generates a $15 \mathrm{~V}, 75 \mathrm{kHz}$ signal. The reason to have independent drivers is due to the motor's capacitance that modifies the supplied-signal by reducing its effective voltage and by reshaping its required square wave-form.

When too many motors are connected in parallel to the same driver-output, the total capacitance increases considerably, making the supplied signal to be so distorted that it can no longer comply with the $15 \mathrm{~V}$, $75 \mathrm{kHz}$ requirement, causing an unreliable performance of the motors (low speed and thrust or even no movement) [23]. Figure 15b illustrates the degradation of the wave as more motors are supplied by the same output-signal.

\subsection{Power source}

As aforementioned, each taxel is optimally driven at $15 \mathrm{~V}$. At this operation point, it consumes $10.8 \mathrm{~mA}$. Thus, the maximum current required from the power source is when all 60 taxels are activated simultaneously; that is $648 \mathrm{~mA}$ plus some current consumed by the electronic drive. Experimental measurements show that a maximum current of $800 \mathrm{~mA}$ (or $12 \mathrm{~W}$ ) is required from the power source to drive the Braille terminal.

As on-board power supply, we have incorporated a $18 \mathrm{~V}, 1 \mathrm{Ah}$ rechargeable Ni-Cd battery pack that ensures a $1.5 \mathrm{~h}$ continuous operation of the terminal. Moreover, the terminal is equipped with an AC to DC converter to receive the $\mathrm{AC}$ input voltage from a wall power plug. 


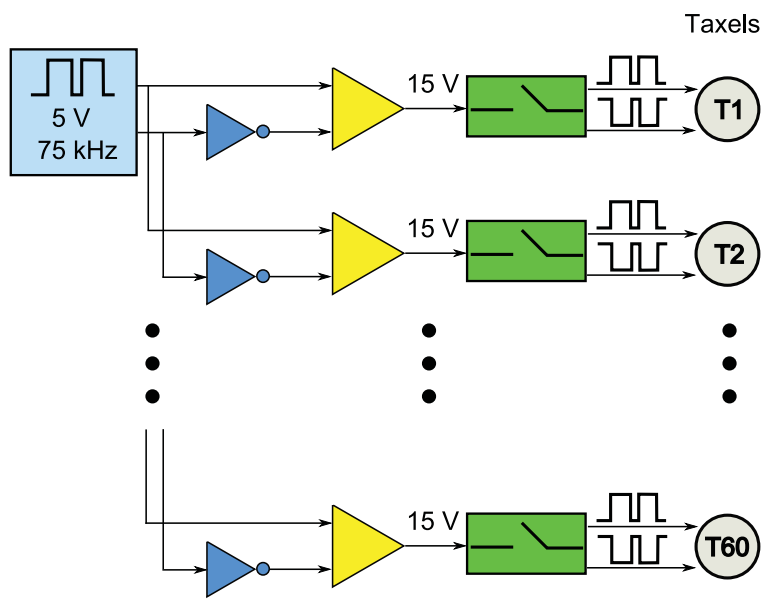

(a) General diagram of the electronic drive.

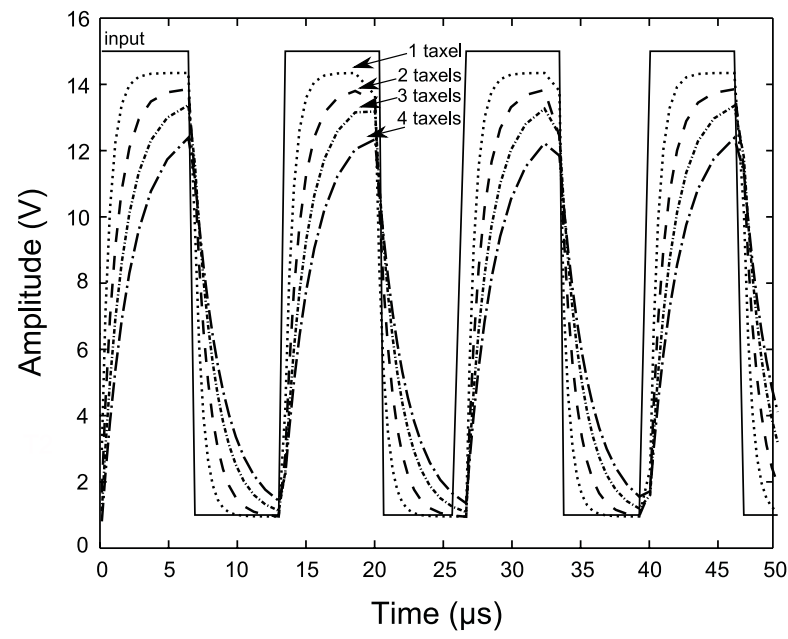

(b) Reduction of the effective voltage according to the number of taxels actuated.

Fig. 15. Electronic drive for the Braille terminal.

\subsection{Processing unit}

The TactoBook's processing unit was developed using the TS-7500 embedded computer module [21]. The TS-7500 features two main logic elements: an ARM922 based CNS2132 processor and a XP2 FPGA Lattice.

With the Linux Angstrom operative system (OS) running on the CNS2132 processor, the TS-7500 is a powerful and flexible solution for embedded applications. For this application, the OS is installed in a SD card and boots Linux 2.6.18 kernel.

Figure 16a shows the processing unit architecture. To control the TactoBook, the embedded system creates a driver that generates a kernel-controlled external device named /dev/tactobook. When the TactoBook software communicates with the TactoBook terminal, the kernel of the embedded system conditions first the information through this driver. Once conditioned, data flows from the processor to the internal registers using a Serial Peripheral Interface (SPI) bus and the Wishbone protocol. It is in these registers where the code that generates the Braille terminal's signals is located. Finally, these signals are delivered by the embedded system, transferred to the electronic drive, and addressed to the piezoelectric taxels.

One of the key features of the TactoBook software is that it includes $u d e v$, the device manager for the Linux 2.6 kernel series. Udev is responsible for identifying and managing the external devices plugged to the system. Basically, udev compares the plugged devices to a series of rules that indicate the action to be performed [12].

For the TactoBook, a rule indicating to execute the TactoBook software is added to udev when detecting a USB memory drive (Fig. 16b). The first task performed by the software is to prompt the user to select a Brailleencrypted file in the memory drive. Files found on the memory drive are sequentially displayed on the Braille terminal so that the user can select one.

The program splits then the text contained in the selected file into chapters, sections, paragraphs, and lines allowing the user to select the reading start point. As in file selection, these options are sequentially displayed on the Braille terminal: first chapter, then section, then paragraph, and finally line.

While reading, the user is able to navigate among the text using the buttons in the Braille terminal. In this first version, the user is able to go word forward/backward and return to the reading start point.

\subsection{Ergonomics}

In collaboration with ergonomists and industrial designers, an ergonomic enclosure has been designed for the Braille terminal to ensure comfort, easier adoption, and a higher user acceptance. 


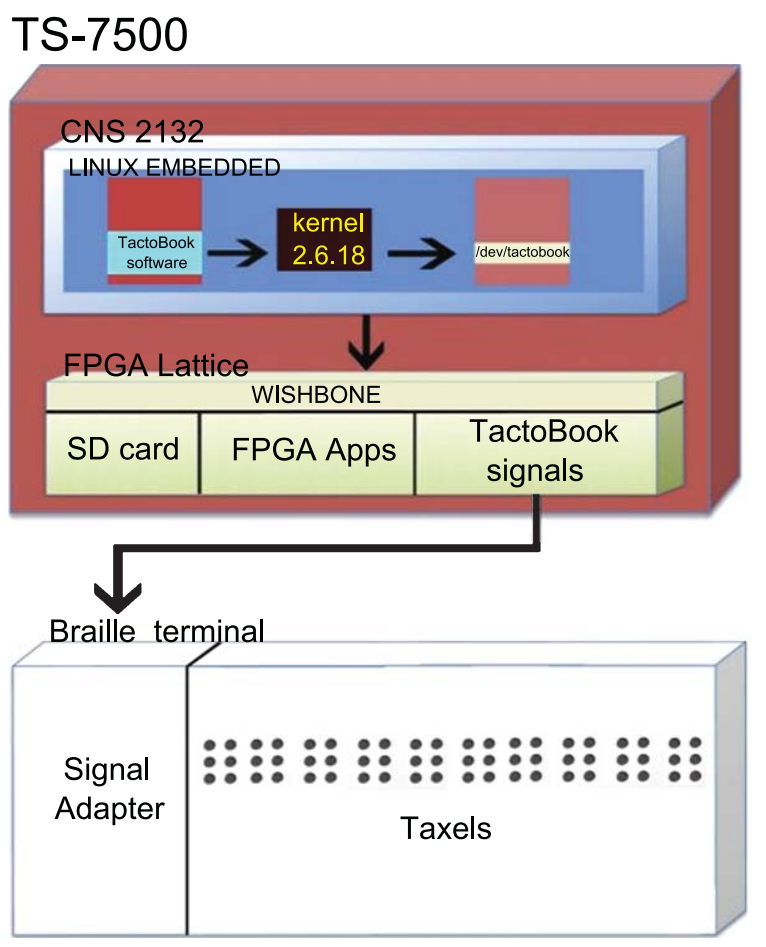

(a) Processing unit.

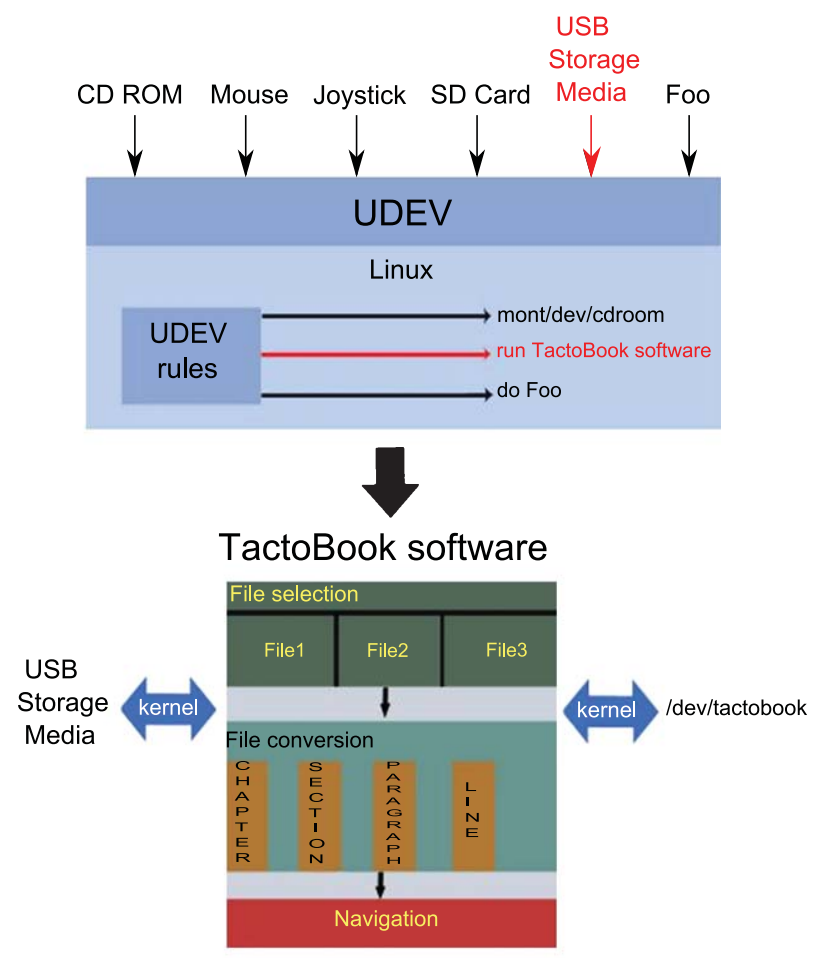

(b) Detection of external devices and navigation among files and text. Foo is the Linux word for "any" (e.g. Foo device is any device, do Foo is do anything).

Fig. 16. The TactoBook's Linux-based processing unit and file/text-navigation structure.

The central ergonomic concept is based on how people will hold the Braille terminal. It is evident that the user will need both hands: one for reading and one for holding the device. However, the holding hand is the key for user acceptance as it is the one that eventually gets tired. If the user does not find a comfortable way to hold the Braille terminal, he will stop using the device after a short time.

Figure 17a shows the depart ergonomic concept. Here, the Braille terminal is held with both palm and forearm to distribute its mass and avoid wrist fatigue. Figure $17 \mathrm{~b}$ shows a schematic drawing of the enclosure envisaged. This design encompasses several ergonomic features:

(1) Bright color for low-vision users,

(2) Big buttons to ease their identification,

(3) Support for the reading fingers, and

(4) Secure neck strap.

Figure $17 \mathrm{c}$ shows the first prototype of ergonomic enclosure developed based on the schematic design of
Fig. 17b. This prototype includes a base as well to recharge the terminal's battery. Figure $17 \mathrm{~d}$ illustrates the desired reading process. Note how the Braille terminal is comfortably held and secured with the user non-reading hand.

Future work expects to integrate the prototype of Fig. 13b with this ergonomic design.

\section{Conclusions and future work}

This paper has presented a novel concept of reading assistive device: the TactoBook, a system which aim is to make eBooks accessible to the blind. The fundamental idea is to translate eBooks to Braille using standard computational resources and to store them as files in a USB memory drive, which is later inserted and reproduced in a compact, lightweight, and highlyportable Braille terminal. This way, Braille readers can access published information immediately and store multiple eBooks in the same device without carrying the burdensome tactile print versions. 


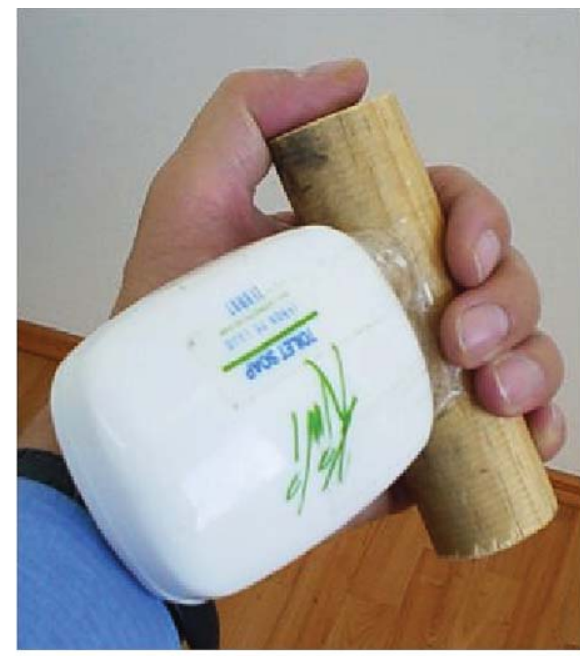

(a) Depart concept.

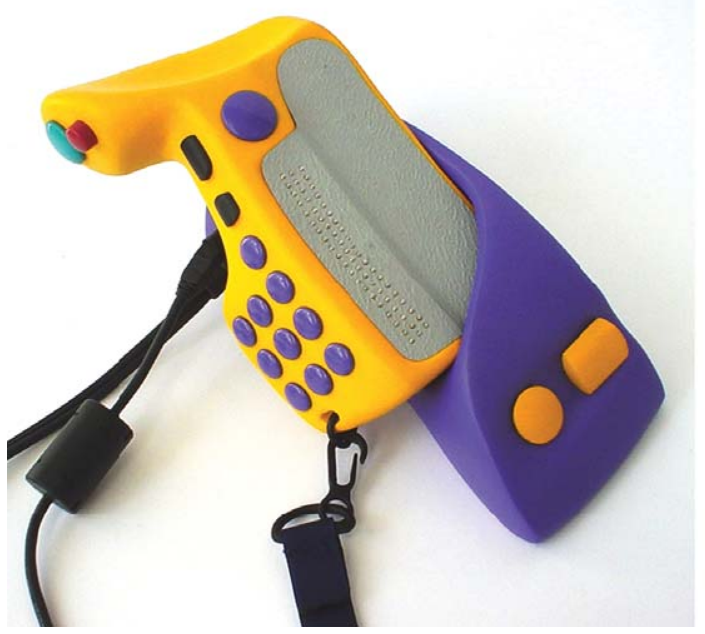

(c) Fist prototype.

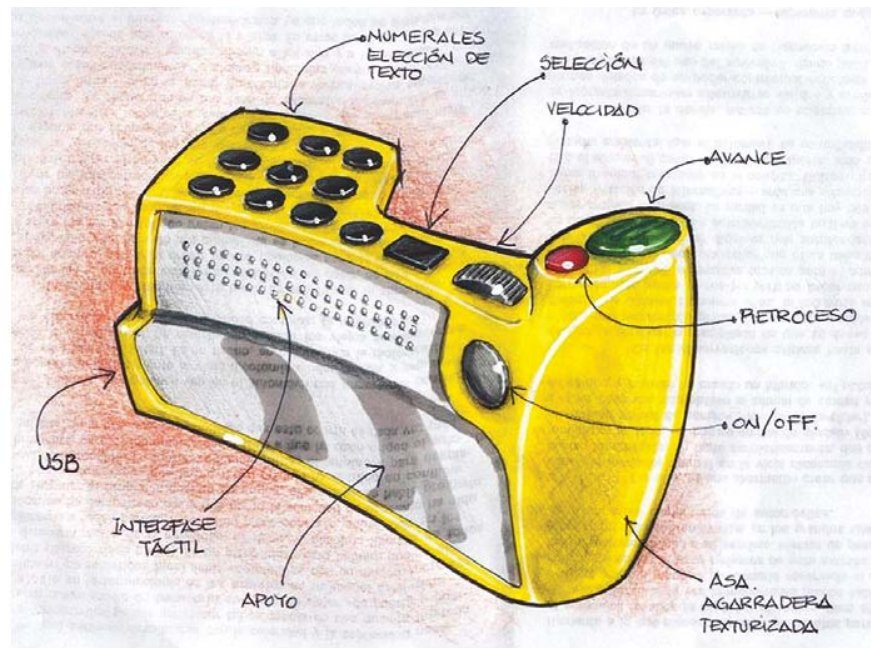

(b) Schematic design.

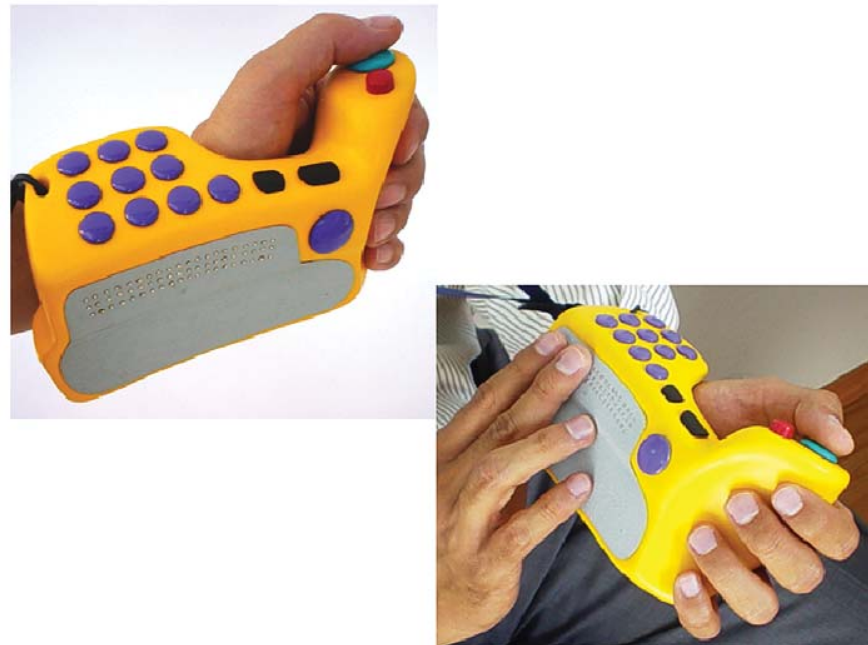

(d) Intended usage.

Fig. 17. Concept and implementation of an ergonomic enclosure for the TactoBook Braille terminal.

Main parts of the system such as Braille translator and tactile terminal have been introduced and technically overviewed. In particular, this paper presented a detailed characterization and experimental evaluation of a piezoelectric ultrasonic linear motor. This motor exhibits significant advantages compared to usual electromagnetic motors: size, compactness, simple structure, and variable stroke. These features make it interesting for a wide number of applications, particularly at the meso/micro scale. This paper discussed its design, performance, and implementation for tactile actuator (taxel) in highly-portable tactile terminals.
A first prototype of Braille terminal has been developed together with its electronic drive, processing unit and ergonomic concept. In particular, the onboard processing unit consists of a Linux-based simple yet powerful embedded system that allows navigation among and along Braille files. Current work with the processing unit includes providing an acoustic feedback to the user whenever he desires to know his current location at the file or whenever the system reports a miss-functional behavior, for instance, when the memory-storage device is not properly connected.

Future work perspectives include: 1) evaluation of the TactoBook's performance with blind Braille 
readers, and 2) the integration of the Braille terminal prototype with its ergonomic concept.

\section{Acknowledgments}

This work was fully financed by CEPii (Centro Panamericano de Investigación e Innovación - Mexico). The authors would like to thank Luis-Arturo Méndez for his valuable help with the ergonomic aspects of this project.

\section{References}

[1] J. Anderson, Guidelines for indexes and related information retrieval devices, NISO Technical Report 2 (1997), 21-34.

[2] J. Brabyn, K. Seelman and S. Panchang, Aids for people who are blind or visually impaired. In: R. Cooper, H. Ohnabe, D. Hobson, Eds. An introduction to rehabilitation engineering, Taylor \& Francis, 2007, pp. 287-313.

[3] Braille Institute of America. Updated information available at: http://www.brailleinstitute.org/ (accessed January 2012).

[4] Duxbury Systems Inc. Updated information available at: http://www.duxburysystems.com/ (accessed January 2012).

[5] Enabling Technologies Inc. Updated information available at: http://www.brailler.com/ (accessed January 2012).

[6] H. Fischer, B. Neisius and R. Trapp, Tactile feedback for endoscopic surgery. In: R. Satava, et al. Eds., Interactive Technology and the New Paradigm for Healthcare, IOS Press, 1995, pp. 114-117.

[7] M. Goldfarb and N. Celanovic, Modeling piezoelectric stack actuators for control of micromanipulation, IEEE Control Systems Magazine 17 (1997), 69-79.

[8] H. Hernandez, E. Preza and R. Velazquez, Characterization of a piezoelectric ultrasonic linear motor for Braille displays, Proceedings of IEEE Electronics, Robotics and Automotive Mechanics Conference, Cuernavaca Mexico, 2009, pp. 402-407.

[9] HumanWare. Updated information available at: www. humanware.com (accessed January 2012).

[10] H. Ko, S. Kim, S. Borodinas, P. Vasiljev, C. Kang and S. Yoon, A novel tiny ultrasonic linear motor using the radial mode of a bimorph, Sensors and Actuators A 125 (2006), 477-481.

[11] M. Konyo, S. Tadokoro and T. Takamori, Artificial tactile feel display using soft gel actuators, Proceedings of IEEE International Conference on Robotics and Automation, San Francisco, CA, USA, 2000, pp. 3416-3421.

[12] G. Kroah-Hartman, Udev - A userspace implementation of devfs, Proceedings of the Linux Symposium, Ottawa, Canada 2003, pp. 263-271.
[13] J. Lee and S. Lucyszyn, A micromachined refreshable Braille cell, Journal of Microelectromechanical Systems 14 (2005), 673-682.

[14] J. Loomis, On the tangibility of letters and Braille, Perception and Psychophysics 29(1) (1981), 37-46.

[15] M. Nakatani, H. Kajimoto, D. Sekiguchi, N. Kawakami and S. Tachi, 3D form display with shape memory alloy, Proceedings of International Conference on Artificial Reality and Telexistence, Tokyo, Japan, 2003, pp. 179-184.

[16] NCTD - National Center for Tactile Diagrams. Updated information available at: http://www.rnib.org.uk/ (accessed January 2012)

[17] Piezoelectric Technology Co. Sinnae Technotown, Seoul, Korea, 131863. Updated information available at: http://www.piezo-tech.com/eng/product/ultrasonic.asp (accessed January 2012).

[18] Quantum Technology Updated information available at: http://www.quantumtechnology.com.au/ (accessed January 2012).

[19] I. Summers and C. Chanter, A broadband tactile array on the fingertip, Journal of the Acoustical Society of America 112 (2002), 2118-2126.

[20] P. Taylor, D. Pollet, A. Hosseini and C. Varley, Advances in an electrorheological fluid based tactile array, Displays $\mathbf{1 8}$ (1998), 135-141.

[21] Technologic Systems. Updated information available at: http://www.embeddedarm.com/ (accessed January 2012).

[22] R. Velazquez and E. Pissaloux, Tactile displays in humanmachine interaction: Four case studies, The International Journal of Virtual Reality 7(2) (2008), 51-58.

[23] R. Velazquez, E. Preza and H. Hernandez, Making eBooks accessible to blind Braille readers, Proceedings of IEEE International Workshop on Haptic Audio Visual Environments and Games, Ottawa, Canada, 2008, pp. 25-29.

[24] F. Vidal, M. Madueno and R. Navas, Thermopneumatic actuator for tactile displays and smart actuation circuitry, Proceedings of SPIE International Symposium on Microtechnologies for the New Millenium, Sevilla, Spain, 2002, pp. 484-492.

[25] C. Wagner, S. Lederman and R. Howe, Design and performance of a tactile shape display using RC servomotors, Proceedings of International Symposium on Haptic Interfaces for Virtual Environments and Teleoperator Systems, Orlando, FL, USA, 2002, pp. 354-355.

[26] WHO - World Health Organization. 2011. Visual impairment and blindness - Fact Sheet No 282. Available from: http://www.who.int/mediacentre/factsheets/fs282/en/ (accessed January 2012).

[27] F.H. Yeh, H.S. Tsay and S.H. Liang, Applied CAD and ANFIS to the Chinese Braille display optimization, Displays 24 (2003), 213-222. 

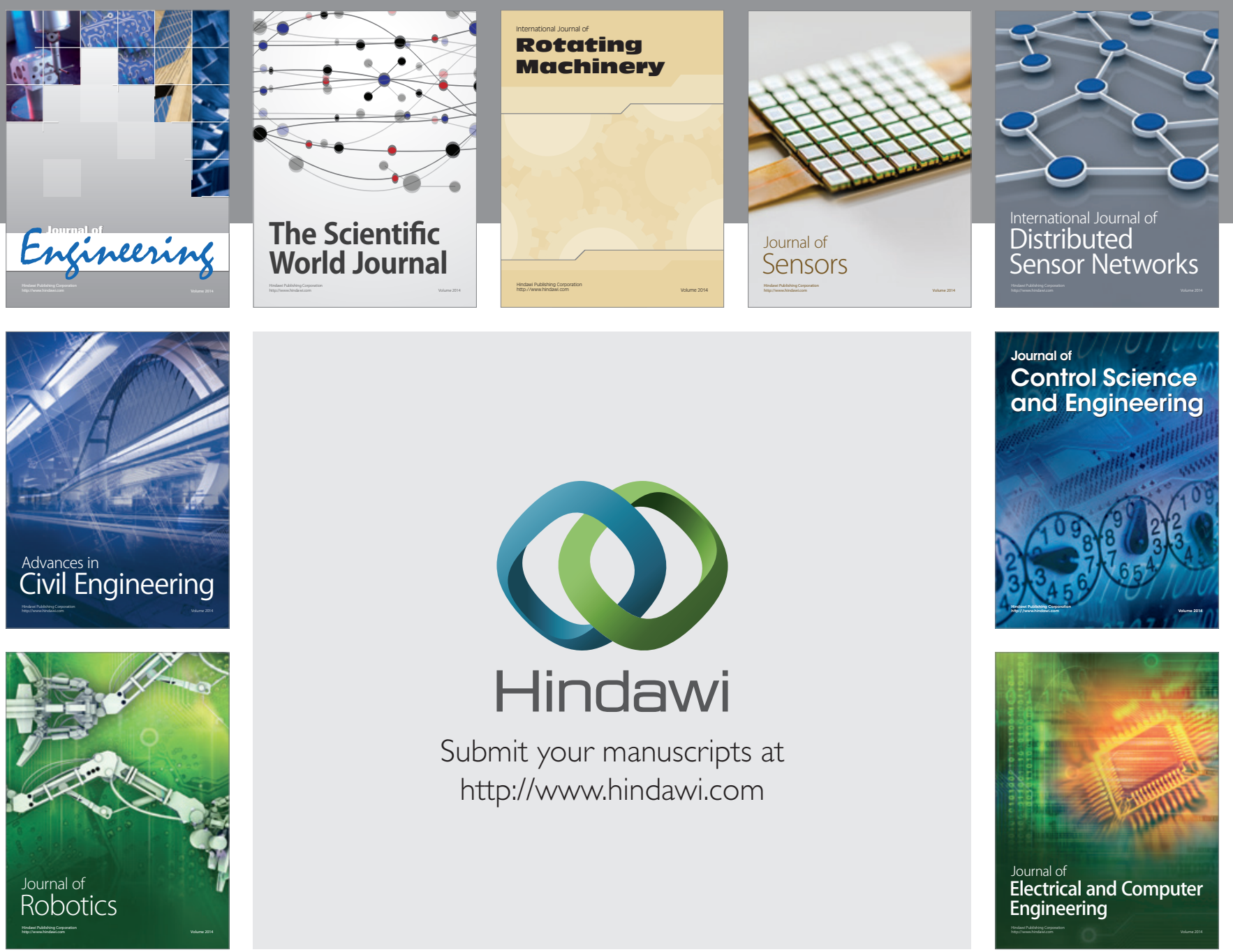

Submit your manuscripts at

http://www.hindawi.com
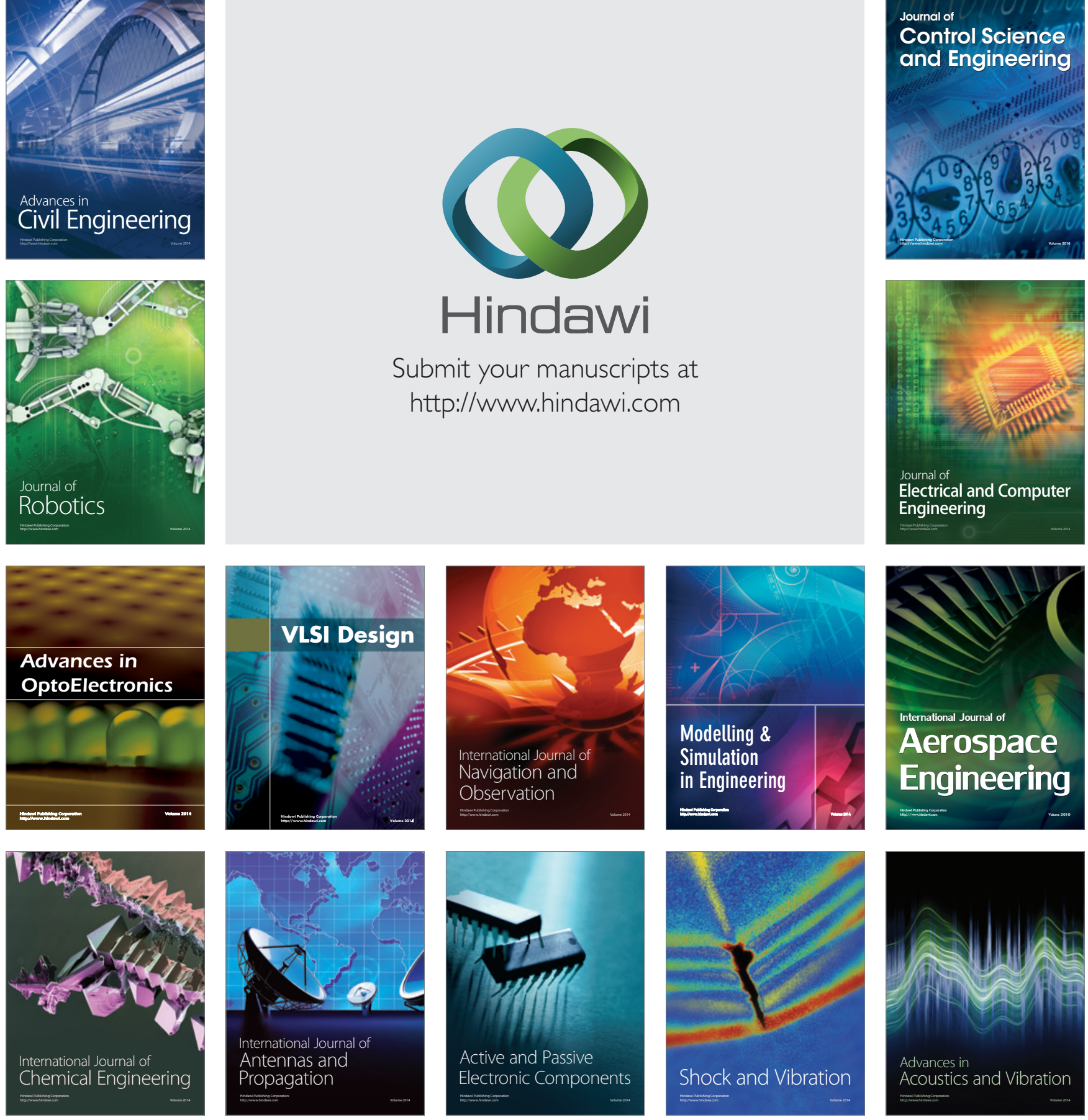